\title{
As-built new Mayall telescope top end for the DESI project
}

Gaston Gutierrez, Robert W. Besuner, David Brooks, Peter Doel, Brenna Flaugher, et al.

Gaston Gutierrez, Robert W. Besuner, David Brooks, Peter Doel, Brenna Flaugher, Donald Friend, Giuseppe Gallo, Andrew Stefanik, Gregory Tarle, "As-built new Mayall telescope top end for the DESI project," Proc. SPIE 10702, Ground-based and Airborne Instrumentation for Astronomy VII, 107027Y (10 July 2018); doi: 10.1117/12.2312728

Event: SPIE Astronomical Telescopes + Instrumentation, 2018, Austin, Texas, United States 


\title{
As-built new Mayall telescope top end for the DESI project
}

\author{
Gaston Gutierrez ${ }^{\mathrm{a}}$, Robert W. Besuner ${ }^{\mathrm{b}}$, David Brooks ${ }^{\mathrm{c}}$, Peter Doel ${ }^{\mathrm{c}}$, Brenna Flaugher ${ }^{\mathrm{a}}$, \\ Donald Friend ${ }^{\mathrm{a}}$, Giuseppe Gallo ${ }^{\mathrm{a}}$, Andrew Stefanik ${ }^{\mathrm{a}}$, and Gregory Tarle ${ }^{\mathrm{d}}$ \\ ${ }^{a}$ Fermi National Accelerator Laboratory, Batavia, IL 60510, USA \\ ${ }^{b}$ University of California Berkeley, Space Science Laboratory, 1 Cyclotron Road, Berkeley, CA \\ 94720, USA \\ ${ }^{\mathrm{c} A s t r o p h y s i c s ~ G r o u p, ~ U n i v e r s i t y ~ C o l l e g e ~ L o n d o n, ~ G o w e r ~ S t r e e t, ~ L o n d o n ~ W C 1 E ~ 6 B T, ~ U K ~}$ \\ ${ }^{d}$ Physics Department, University of Michigan Ann Arbor, MI 48109, USA
}

\begin{abstract}
The Dark Energy Spectroscopic Instrument (DESI) is under construction to measure the expansion history of the Universe using the Baryon Acoustic Oscillation technique. The spectra of 35 million galaxies and quasars over $14000 \mathrm{sq}$ deg will be measured during the life of the experiment. A new prime focus corrector for the KPNO Mayall telescope will deliver light to 5000 fiber optic positioners. The fibers in turn feed ten broadband spectrographs. We will present an overview of the mechanical structure that sits atop the Mayall Serrurier trusses and supports the six lenses, the Atmospheric Dispersion Compensator (ADC) rotator and the Focal Plane Assembly. This mechanical structure has already been built, we will describe the main technical requirements and challenges during the construction.
\end{abstract}

Keywords: Prime Focus, Mayall Telescope, Cosmic Acceleration, Dark Energy

\section{INTRODUCTION}

The Dark Energy Survey Instrument (DESI) will measure the expansion history of the universe with unprecedented precision. Over the course of a five-year survey, DESI will measure the spectra of 35 million galaxies and quasars from the present to beyond redshift three and use the Baryon Acoustic Oscillation (BAO) technique to derive cosmological parameters. The precision of DESI's expected measurement of the dark energy equation of state is such that DESI meets the criteria for a Stage IV dark energy experiment.

Figure 1 shows a model of the Mayall telescope with many of the DESI hardware components. The components described in this writeup include the New Upper Ring, Spiders and Cage which sit atop the old Mayall Serrurier trusses, the hexapod system that is supported by the Cage and the Corrector Barrel system that supports the six lenses, the Focal Plane Assembly and the ADC rotator system. For a summary on the entire Mayall upgrade for the DESI experiment see "Overview of the dark energy spectroscopic instrument" ${ }^{1}$ (this Proceedings) and references therein.

\section{CORRECTOR BARREL}

The DESI prime focus corrector has a $3.2 \mathrm{deg}$ diameter field of view with six 0.8 to $1.14-\mathrm{m}$ diameter lenses ${ }^{2}$ (see Figure 2). These include a two-element Atmospheric Dispersion Compensator (ADC). The average focal ratio is $f / 3.86$ and the average plate scale on the aspheric focal surface is $254.8 \mathrm{~mm} / \mathrm{deg}$. The six lenses are mounted in cells that are held in position within a steel corrector barrel assembly. A six degree-of-freedom hexapod maintains the alignment of the corrector and focal plane system.

Three conceptual guidelines were followed in the design of the corrector barrel, 1) to minimize the mass and barrel diameter, without affecting the barrel's deflections and overall stiffness, we used a single shell design and minimized the number of ribs and gussets, 2) to reduce the barrel deflections we minimized the number of barrel

Further author information: (Send correspondence to G.G.)

G.G.: E-mail: gaston@fnal.gov, Telephone: 16308404107

Ground-based and Airborne Instrumentation for Astronomy VII, edited by Christopher J. Evans, Luc Simard, Hideki Takami, Proc. of SPIE Vol. 10702, 107027Y · C 2018 SPIE · CCC code: 0277-786X/18/\$18 · doi: 10.1117/12.2312728 


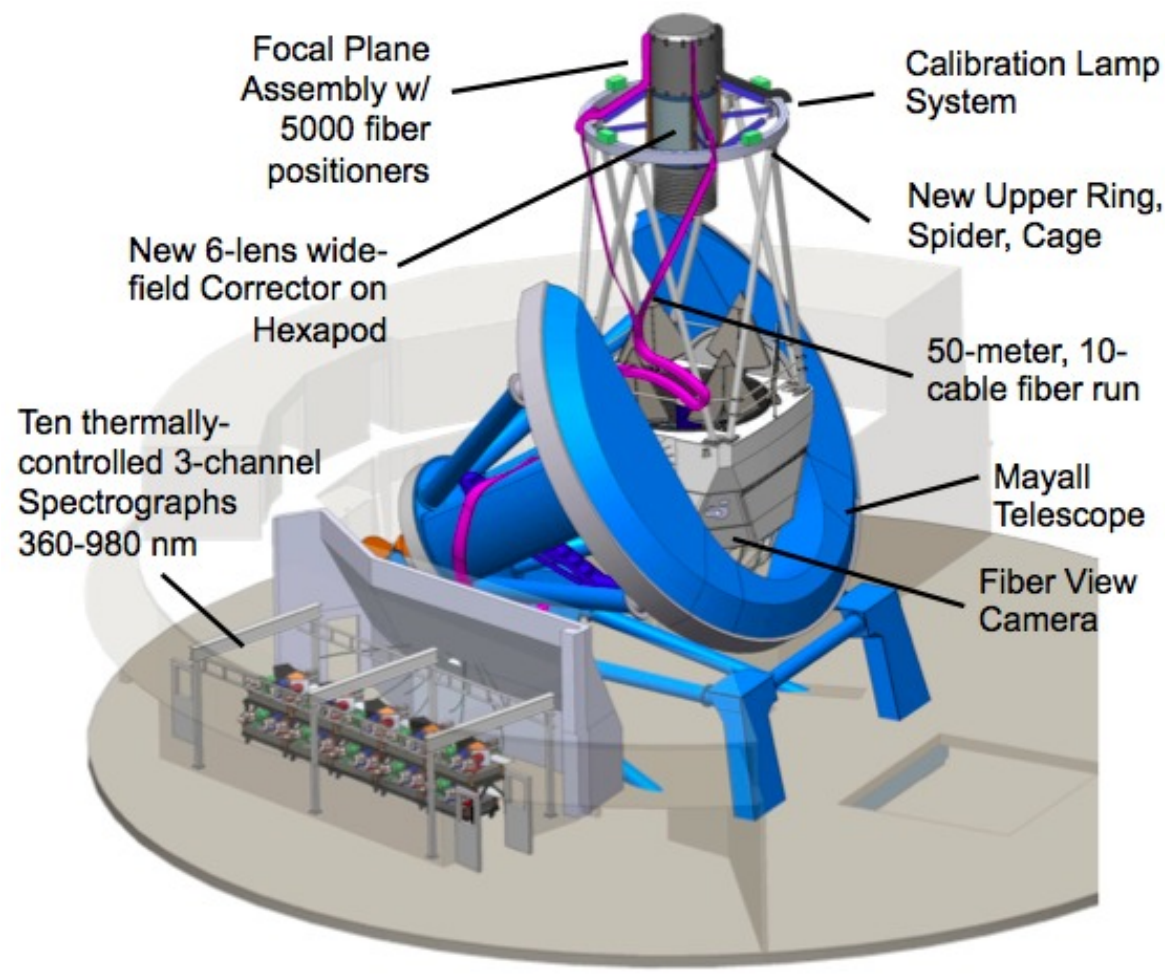

Figure 1. Model of the Mayall telescope with the addition of DESI hardware. The calibration lamp system is mounted to the new upper ring. The ring, spider, and cage supports the new corrector via a hexapod. The Focal Plane System is mounted to the end of the Corrector. Ten fiber cables, each with over 500 fibers, run from the Focal Plane System, along the telescope structure, and into a thermally-controlled room within the Mayall Large Coudé Room. A slithead at the end of each fiber cable directs light into a spectrograph, which disperses the light onto three detectors per spectra. All of the instrument is controlled with the Instrument Control System. The data are rapidly transferred offsite where they are processed through a custom pipeline.

segments, and 3) to minimize cost and risk we used a hexapod design similar to DECam. The detail rationale for each of the previous guidelines was discussed "Thoughts on minimizing deflections in the MS-DESI barrel design" 3 and "Final design of the DESI corrector barrel for the ECHO22 optics." 4

The corrector barrel has three main sections that support the optics. Each of these sections supports two lenses, so each lens can be mounted from each side of the corresponding section. The Front section supports the $\mathrm{C} 1$ and $\mathrm{C} 2$ lenses, the After section supports the C3 and C4 lenses, and the Middle section supports the $\mathrm{ADC}$ lenses $\mathrm{ADC} 1$ and $\mathrm{ADC} 2$. The $\mathrm{ADC}$ rotation mechanism, based on a custom bearing driven by harmonic drive motors, attaches to the Middle section. The hexapod attaches to the After section of the barrel and to the cage. Its purpose is to adjust the location of the corrector and focal plane system as needed to maintain optical alignment. The barrel also contains a Shroud designed to stand the weight of the entire corrector and protect C1 during the construction phase, and a Focal Plane Adapter (FPD) that supports the Focal Plane Assembly (FPA). The lenses are mounted in cells, that accommodate the differences in the coefficient of thermal expansion (CTE) between the lens materials and the steel corrector barrel, as well as maintaining alignment over the full range of zenith angles. This structure (without the ADC rotator) is shown in Figure 2.

\subsection{Requirements, Construction and as-built errors}

Table 1 shows the main requirements that applied to the construction of the corrector barrel, the Cage and Upper Ring. The static tolerance, shown in columns 2 and 3 in the table, refer to the maximum errors introduced in the process of aligning the cells in the barrel. The dynamic tolerances, shown in columns 4 and 5 , refer to the 

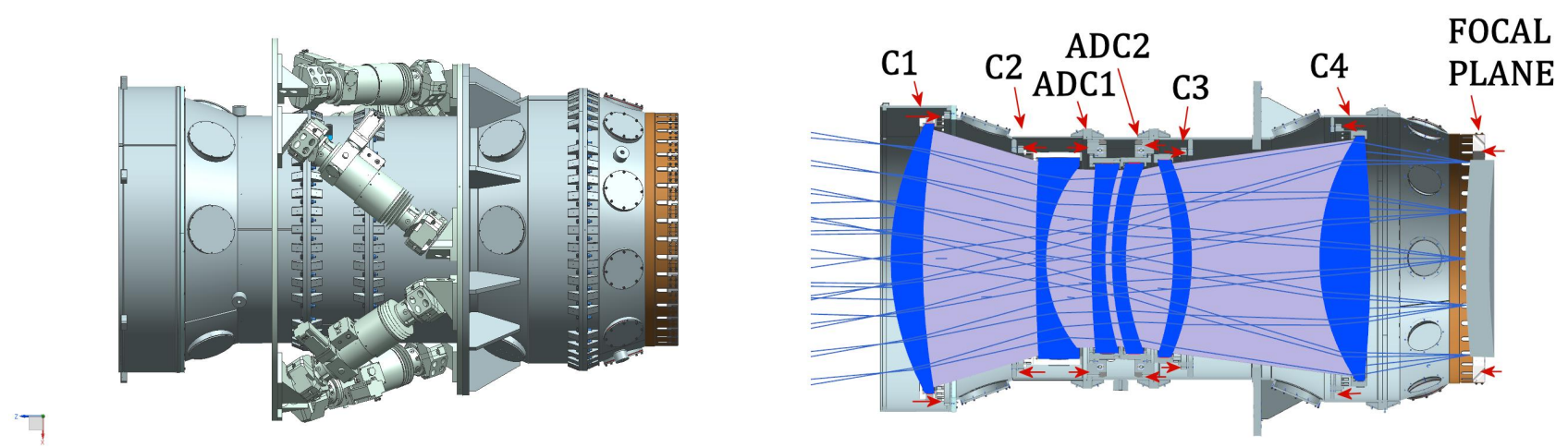

Figure 2. DESI corrector barrel (left pictures) showing the five sections of the corrector barrel and the hexapod, from left to right we have the Shroud, Front Section, Middle Section, After Section and the Focal Plane Adaptor or FPD. The picture on the right shows a section view of the corrector barrel displaying how the $\mathrm{C} 1, \mathrm{C} 2$, C3 and C4 lenses and the two Atmospheric Dispersion Correctors ADC1 and ADC2 are positioned inside the barrel. The red arrows show the direction in which the lenses attach to the barrel. The light purple volume is the keep-out volume for the light created by joining the clear apertures for each lens.

barrel deflections plus the errors introduced by the ADC rotation mechanism. These performance requirements apply over a range of Zenith angles from 0 to 60 degrees.

The specifications of the cell alignment with respect to the barrel are $\pm 20 \mu \mathrm{m}$ for the lateral displacement and about $20 \mu \mathrm{m}$ at the edge of the lenses for the tip/tilt angles. The second part of Table 1 contains the barrel lens cells axial tolerances, barrel out-of-roundness, air and light tightness, barrel material, weight limits for the barrel and the cells, and the assembly/disassembly repeatability requirements for the barrel pieces. Finally the last part of Table 1 specifies the flatness and parallelism of the barrel flanges.

Table 1. Alignment and deflection requirements on Lens cells and Focal Plane, weight requirements on Barrel/Cage and Cells, and assembly/disassembly repeatability requirements.

\begin{tabular}{|c|c|c|c|c|}
\hline \multirow[b]{2}{*}{ Element } & \multicolumn{2}{|c|}{ Static tolerances } & \multicolumn{2}{|c|}{ Dynamic tolerances } \\
\hline & $\begin{array}{l}\text { Lateral } \\
( \pm \mu \mathrm{m})\end{array}$ & $\begin{array}{c}\text { Tilt } \\
( \pm \mu \mathrm{rad})\end{array}$ & $\begin{array}{l}\text { Lateral } \\
( \pm \mu \mathrm{m})\end{array}$ & $\begin{array}{c}\text { Tilt } \\
( \pm \mu \mathrm{rad})\end{array}$ \\
\hline $\mathrm{C} 1$ & 20 & 18 & 50 & 45 \\
\hline $\mathrm{C} 2$ & 20 & 22 & 15 & 53 \\
\hline $\mathrm{ADC} 1$ & 20 & 25 & 75 & 113 \\
\hline $\mathrm{ADC} 2$ & 20 & 25 & 75 & 112 \\
\hline $\mathrm{C} 3$ & 20 & 24 & 45 & 54 \\
\hline $\mathrm{C} 4$ & 20 & 19 & 50 & 14 \\
\hline Focal Plane & 20 & 25 & 40 & 25 \\
\hline Static axial tolerance in all barrel flanges and FPD & \multicolumn{4}{|c|}{$\overline{c \pm 100 \mu \mathrm{m}}$} \\
\hline Flange distortion (circularity) & \multicolumn{4}{|c|}{$50 \mu \mathrm{m}$ peak-to-peak } \\
\hline Air and light tightness & \multicolumn{4}{|c|}{ yes } \\
\hline Barrel material & \multicolumn{4}{|c|}{ Carbon Steel } \\
\hline Telescope Top end Mass & \multicolumn{4}{|c|}{$10,700 \mathrm{Kg}$} \\
\hline Mass of outer ring/spiders/cage/hexapod/barrel & \multicolumn{4}{|c|}{$\leq 7,000 \mathrm{Kg}$} \\
\hline Mass of cells/base rings/spacers & \multicolumn{4}{|c|}{$\leq 560 \mathrm{Kg}$} \\
\hline Barrel assembly/disassembly repeatability & \multicolumn{4}{|c|}{ $\pm 5 \mu \mathrm{m}$ per flange } \\
\hline Flange flatness & \multicolumn{4}{|c|}{$15 \mu \mathrm{m}$} \\
\hline Flange parallelism & \multicolumn{4}{|c|}{$15 \mu \mathrm{m}$} \\
\hline
\end{tabular}


Figure 3 shows pictures of the corrector barrel at different stages of construction. The picture on the left shows the fully assembled barrel in the large Coordinate Measuring Machine (CMM) at Fermilab that was used to do the barrel and lens cells alignment. The picture on the upper right displays the inside of the barrel with all the lens cells. And finally the picture on the lower right shows the barrel Middle section with the bearings use to rotate the ADC lenses and the lens cells. The yellow color is just the paint primer that was used during alignment.
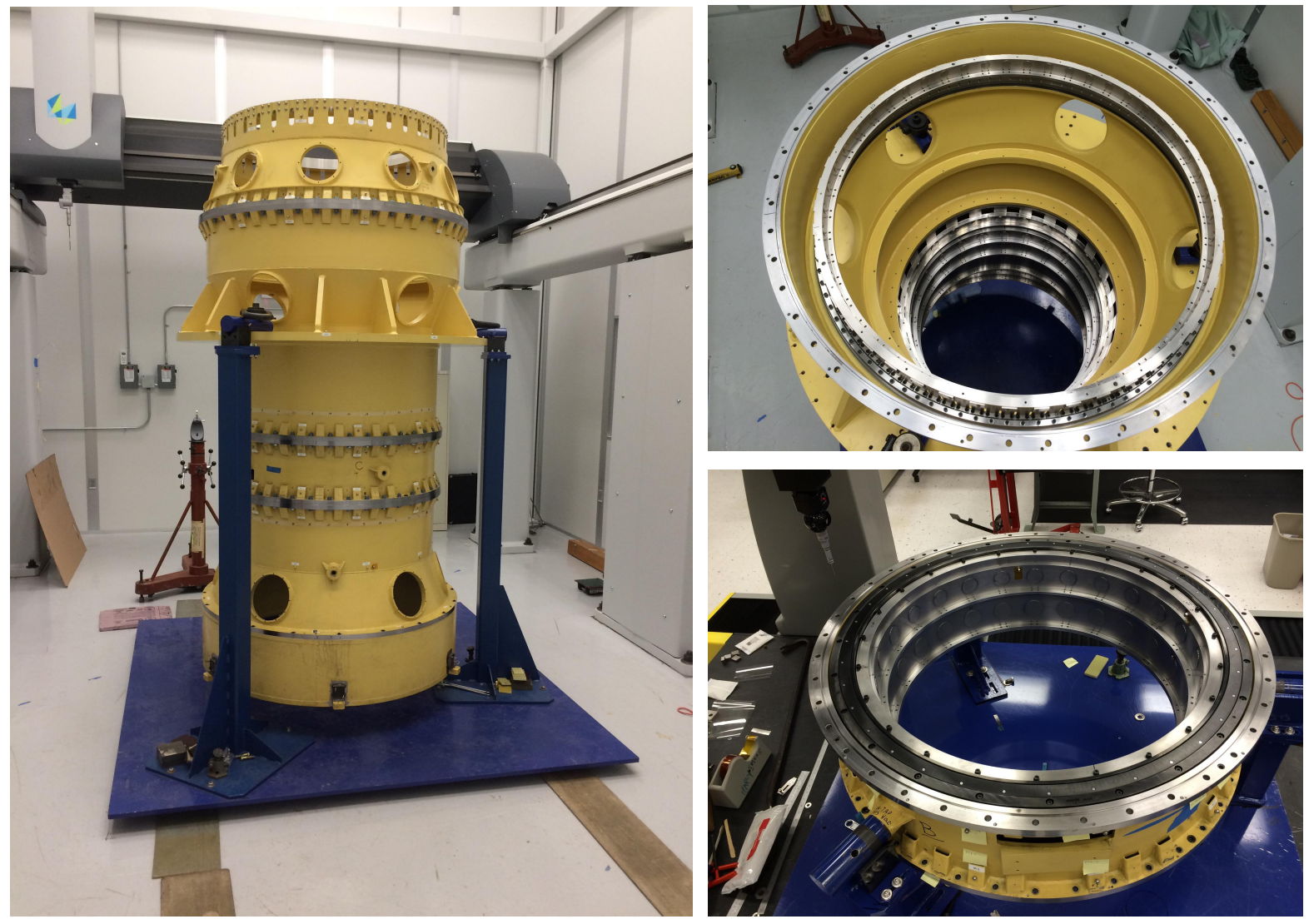

Figure 3. Fully assembled DESI corrector barrel in the Fermilab CMM used for the alignment (left), a view of the lens cells inside the barrel (upper right) and a picture of the Middle section showing the lens cells that support the ADC lenses and the bearings that is used to rotate the lenses (lower right). The yellow color is just the temporary primer, after all the work was finished the barrel was painted with black Aeroglaze Z306.

All the requirements outlined on Table 1 were met. As an example of the measurements performed to check that the corrector barrel met requirements, in Figure 4 we show the position and tip/tilt of the cells after the alignment was finished. All cells were measured at the same time using a large CMM at Fermilab. These plots show the final measurement after the cells were aligned, the barrel was disassembled to pin the lens cells, and then the barrel was reassembled one last time to perform the measurements shown in the plots. The plot on the left shows the center of the $\mathrm{C} 1, \mathrm{C} 2, \mathrm{C} 3$ and $\mathrm{C} 4$ lens cells. The center of rotation of the ADC1 and ADC2 cells was defined as the zero of the coordinate system and is by definition at the center of the plot. The circle shows the $\pm 20 \mu \mathrm{m}$ cell centering requirement plus the $\pm 5 \mu \mathrm{m}$ corresponding to the barrel assemble/disassemble repeatability requirement. The plot on the right of the figure shows the tilt of the lens cells in the directions perpendicular to the barrel axis. The circle corresponds to the $\pm 20 \mu \mathrm{rad}$ requirement.

The corrector barrel is now at the University College London being integrated with the lenses. ${ }^{5}$ 


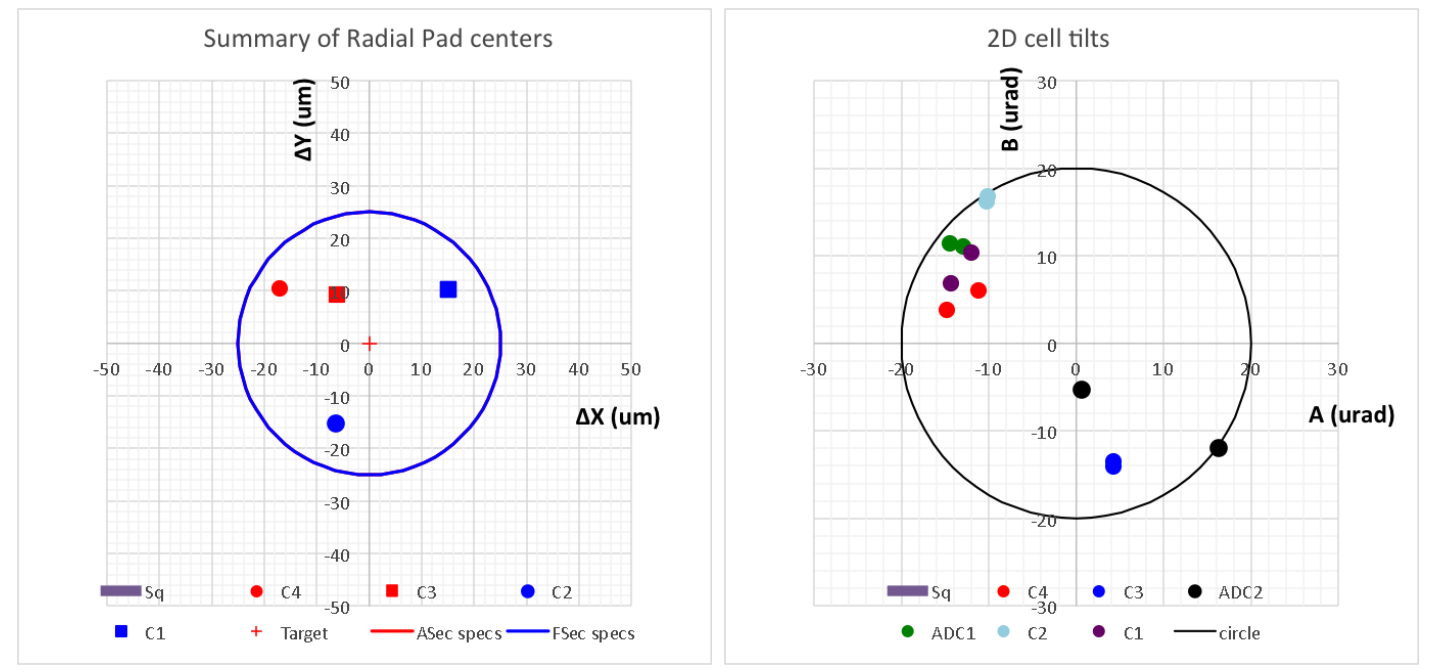

Figure 4. Final lens cell measurements after the pinning of the cells to the barrel. The plot on the left show the center of the $\mathrm{C} 1, \mathrm{C} 2, \mathrm{C} 3$ and $\mathrm{C} 4$ cells relative to the center of rotation of the ADC1 and ADC2 lenses. The plot on the right side shows the tip/tilt of all the lens cells. In both cases the circles show the requirements. The double set of dots in the right plot correspond to measurements performed at two different times and give and idea of the stability of the measurements.

\section{ADC ROTATOR SYSTEM}

The front and back surfaces of the lenses labeled as ADC1 and ADC 2 in Figure 2 are polished with a wedge between them. The wedges act as a prism and the relative orientation of these two prisms allow for the compensation of the atmospheric dispersion. So the two lenses need to be rotated between exposures. Both lenses are part of the corrector barrel Middle section. The cells that hold the ADC lenses are attached to two custom bearings made by KAYDON Bearings (SKF Group Company). These bearings are heavily preloaded to ensure a minimal ran out during rotation. The bearings rotate keeping both the center of the inner race and the motion in and out of the plane of the bearing to the level of $10 \mu \mathrm{m}$. The bearings are rotated by a pinion and gears custom made by Nexen Group Inc. These gears and pinions ran without any backlash and need only a very light coating of grease, which is important given the proximity between the gears and the lenses. The pinions are driven by standard harmonics drives made by the Harmonic Drive LLC. The left picture on Figure 5 shows one of the bearings with the gear segments attached to it. The lower right picture on Figure 3 show the Middle section assembled with both bearings and the lens cells for the ADC1 and ADC2 lenses. The ADC rotator is now at UCL waiting for the full installation and testing with the rest of the corrector barrel and the lenses.

\section{HEXAPOD SYSTEM}

As the telescope moves across the sky the mechanical structure of the telescope deflects beyond the requirements of the DESI experiment. The hexapod is designed to compensate for these mechanical deflections and to keep the corrector correctly positioned relative to the primary mirror at all times. The DESI and the Dark Energy Survey (DES) correctors are similar in size and weight, so to reduce cost and schedule risks DESI decided to use a hexapod design very similar to the DES one. The DESI hexapod was designed and built by A.D.S. International LLC, the same company that built the DES hexapod.

The main specifications for the DESI hexapod are shown in Table 2. The specifications for the resolution are $10 \mu \mathrm{m}$ in the lateral direction, $5 \mu \mathrm{m}$ in the axial direction, $1.5 \mathrm{arcsec}$ in Tip/Tilt and $\pm 3 \operatorname{arcsec}$ in Roll. Tip/Tilt (Roll) refers to rotations around directions perpendicular (along) the axis of the corrector. The hexapod range is $\pm 8 \mathrm{~mm}$ Lateral, $\pm 10 \mathrm{~mm}$ Axial, \pm 250 arcsec Tip/Tilt and \pm 600 arcsec in Roll. The resolution requirements are tighter and the Tip/Tilt range is larger than than the DES equivalent requirements. The left picture in Figure 5 shows the DESI hexapod at A.D.S. International LLC after final assembly and testing. A complete set of tests 

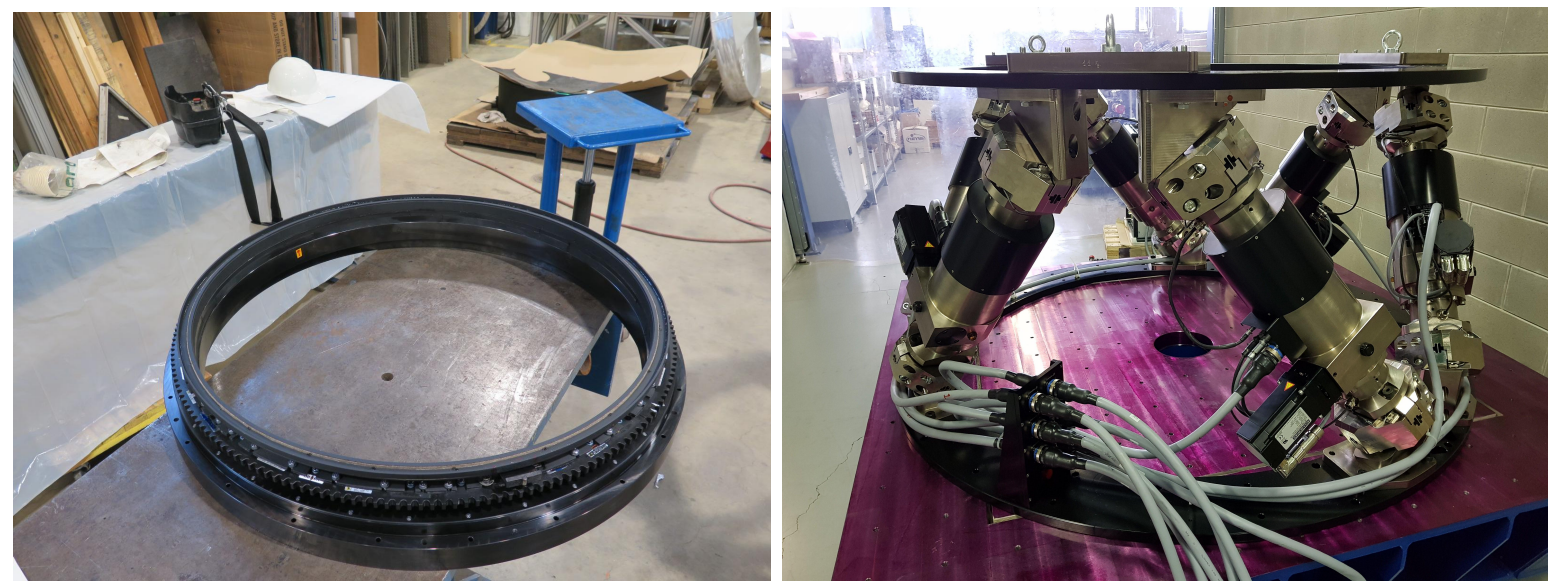

Figure 5. The picture on the left shows one of the two ADC rotator bearing and gear segments. The outer race of the bearings attaches to the Middle section and the inner race to the ADC lens cell. The gear is driven by a harmonic drive mounted to the outside of the Middle section. The picture on the right shows the final assembly of the hexapod at A.D.S International LLC.

on the DESI hexapod performed at A.D.S. showed that the very tight hexapod requirements were met. The hexapod is now at Kitt Peak awaiting installation.

Table 2. Relevant Cage and Hexapod requirements.

\begin{tabular}{lc}
\hline Item & Requirements \\
\hline \hline Cage outer diameter & $1.8 \mathrm{~m}$ \\
\hline Hexapod resolution: & \\
Lateral motion & $10 \mu \mathrm{m}$ \\
Axial motion & $5 \mu \mathrm{m}$ \\
Tip, tilt & $1.5 \mathrm{arcsec}$ \\
Roll & $\pm 3 \mathrm{arcsec}$ \\
Hexapod range: & $\pm 8 \mathrm{~mm}$ \\
Lateral range & $\pm 10 \mathrm{~mm}$ \\
Axial range & $\pm 250 \mathrm{arcsec}$ \\
Tip, tilt range & $\pm 600 \mathrm{arcsec}$ \\
Roll range
\end{tabular}

\section{UPPER RING, VANES AND CAGE}

The top left picture in Figure 6 shows a 3D model of the Upper Ring, the Vanes, the Cage and the Hexapod. The Cage (inner structure in the picture) is composed of three rings: the primary mirror or PM ring that supports the light baffles, the middle on Hexapod Cage ring that supports the hexapod and the focal plane or FP ring that supports the thermal shield for the Focal Plane Assembly. Four rails keep these three rings as a solid unit. Three vanes insert in each of the rails at one end and at the Upper Ring at the other end. The vanes provide adjustment at the Upper Ring end in order to be able to center the Cage with respect to the primary mirror. The Upper Ring holds the entire structure on top of the Serrurier trusses.

For the Cage we selected an open design similar to DES. The main advantage is that it provides easy access to the hexapod and the ADC rotators, the disadvantage is that a middle vane or spider is needed in order to minimize deflections. DESI has no needs for an $\mathrm{f} / 8$ mirror like DECam so the old Mayall flipping ring was 

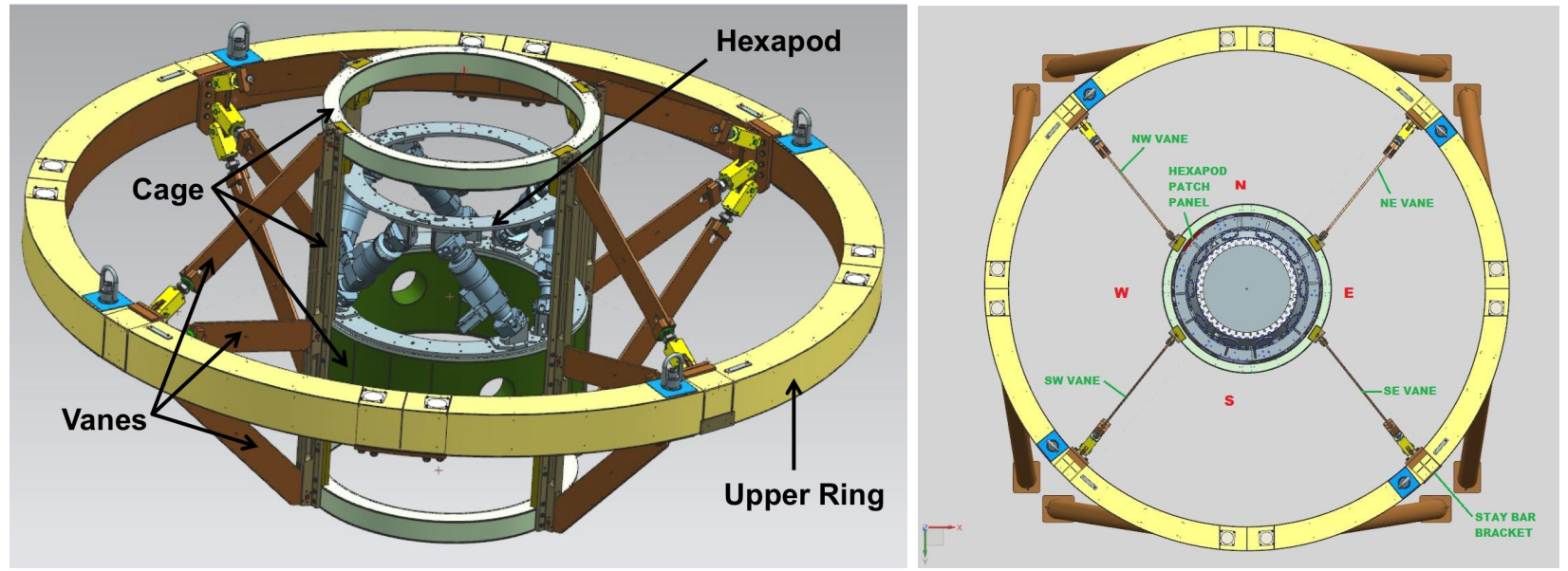

Figure 6. The picture on the left shows a 3D model of the Upper Ring, the Vanes or spiders, the Cage and the hexapod. The picture on the right shows how the vanes insert on the cage's rails.

eliminated and the outer ring was re-designed to keep the total weight of the entire telescope top end replacement at or below the weight of the replaced structure.

The vertical sag of the cage's position with respect to the top end of the Serrurier trusses when the telescope at zenith is required to be less that $0.9 \mathrm{~mm}$ which is $3 \%$ of the hexapod's range, the sag when the telescope is in the horizontal position is required to be less than $1.4 \mathrm{~mm}$ which is $17.5 \%$ of the hexapod's range and the requirement for the tilt of the cage when the telescope is in the horizontal position is required to be less that 70 arcsec which is $28 \%$ of the hexapod's range. This leaves plenty of room in the range of adjustment of the hexapod to compensate for other effects like telescope deflections and alignment errors.

DESI exposures are 20 minutes long and the focal plane has a very large diameter, so the roll of the Cage during exposure is important and is required to be less than $8 \mu \mathrm{m}$ at the edge of the Focal Plane. In order to provide a restoring force that will prevent the cage from rolling the vanes are not inserted at 90 degrees, or evenly spaced, on the cage. The insertion of the vanes was displace by $18 \mathrm{~cm}$ along the circumference of the cage, relative to the even spaced insertion, with pairs of vanes moving in opposite directions. This generates enough restoring force to keep the roll of the cage within specifications. The right picture in Figure 6 shows how the vanes insert on the cage's rails.

The bottom left picture in Figure 7 shows the as-built structure at CAID industries, the company in Tucson that built the Upper Ring, Vanes and Cage. The right picture shows a detail of the Cage and how the Vanes insert in the Rails. The entire structure has been delivered to Kitt Peak and is now waiting for installation.

\section{SUMMARY}

The Dark Energy Spectroscopic Instrument is more than halfway through the construction phase. A number of components have already arrived at the Mayall, and a large number will be delivered before the end of 2018 . The Mayall telescope is presently in the midst of major changes to the top end. The corrector barrel is being integrated with the lenses and the ADC rotator at UCL and will be delivered to Kitt Peak this summer. The Hexapod is at Kitt Peak National Observatory already as well as the Upper Ring, Vanes and Cage (see Figure 8). The installation of all these elements in the Mayall will be done during the summer of 2018.

\section{ACKNOWLEDGMENTS}

This research is supported by the Director, Office of Science, Office of High Energy Physics of the U.S. Department of Energy under Contract No. DEAC0205CH1123, and by the National Energy Research Scientific Computing 

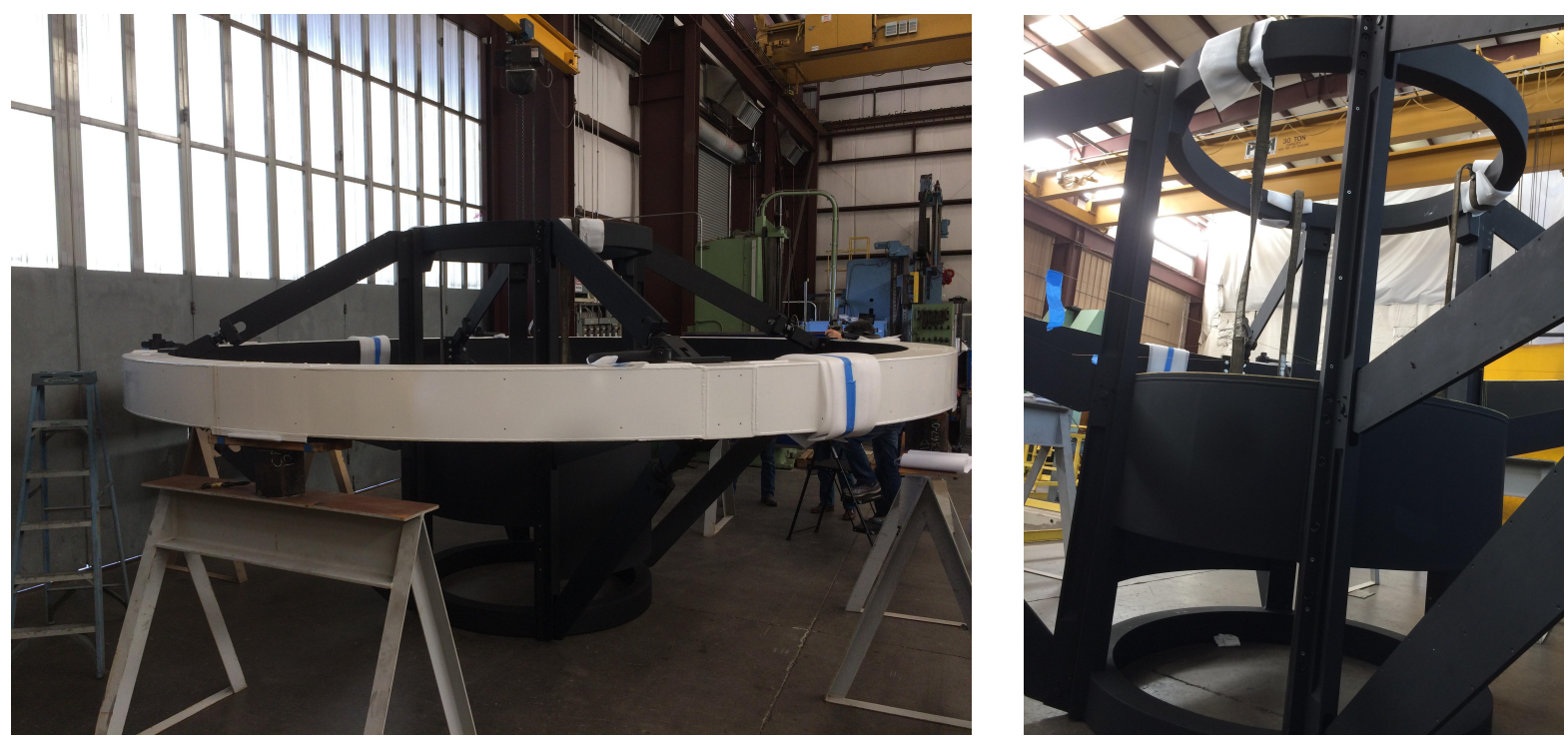

Figure 7. The left picture shows the Upper Ring, Vanes and Cage as-built structure at CAID Industries in Tucson, Arizona. The picture on the right shows a detail of the Cage and how the vanes insert on the cage's rails.

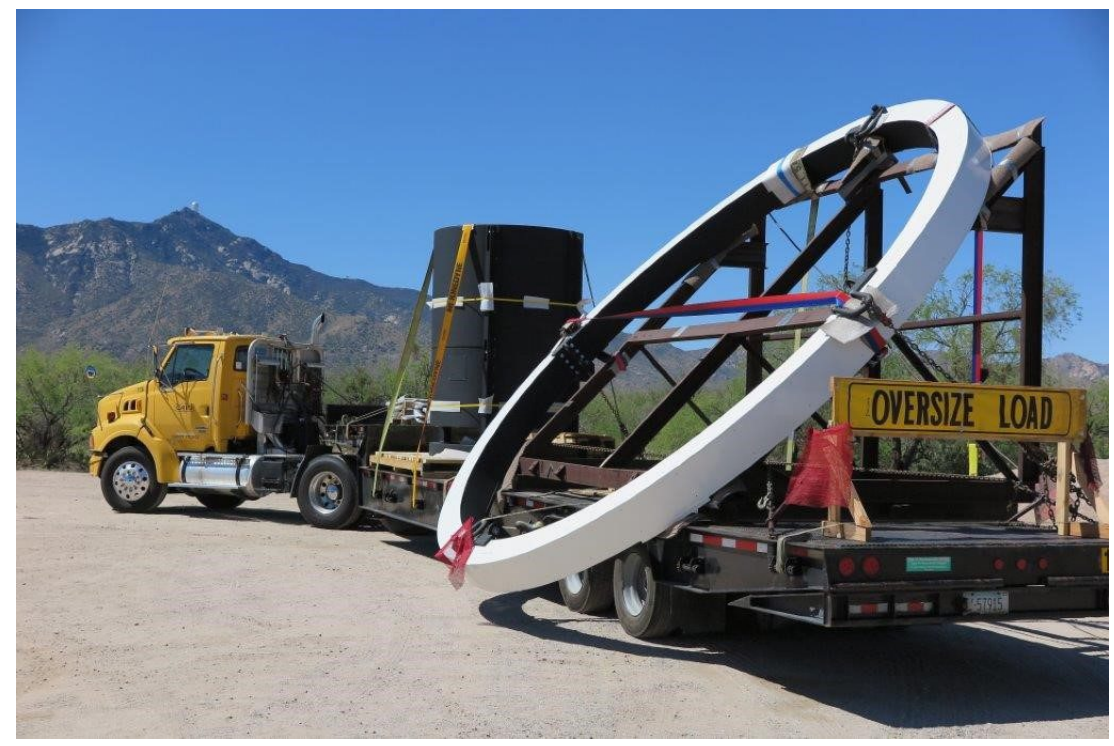

Figure 8. Delivery of the Upper Ring, Vanes and Cage to Kitt Peak National Observatory.

Center, a DOE Office of Science User Facility under the same contract; additional support for DESI is provided by the U.S. National Science Foundation, Division of Astronomical Sciences under Contract No. AST-0950945 to the National Optical Astronomy Observatory; the Science and Technologies Facilities Council of the United Kingdom; the Gordon and Betty Moore Foundation; the Heising-Simons Foundation; the National Council of Science and Technology of Mexico, and by the DESI Member Institutions. The authors are honored to be permitted to conduct astronomical research on Iolkam Duag (Kitt Peak), a mountain with particular significance to the Tohono Oodham Nation. 


\section{REFERENCES}

[1] Martini, P. et al., "Overview of the dark energy spectroscopic instrument," Proc. SPIE 10702, 1070251 (2018).

[2] Miller, T. et al., "Fabrication of the DESI corrector lenses," Proc. SPIE 10706-32, 1070251 (2018).

[3] G. Gutierrez, A. Stefanik and R. Wands, "Thoughts on minimizing deflections in the MS-DESI barrel design." DESI Internal Note, DESI-0330 (2013). Document will be provided upon request.

[4] G. Gallo, G. Gutierrez and A. Stefanik, "Final design of the DESI corrector barrel for the ECHO22 optics." DESI Internal Note, DESI-1162 (2015). Document will be provided upon request.

[5] Doel, A.P.. et al., "The current status of the assembly and alignment of the DESI prime focus corrector," Proc. SPIE 10706-32, 1070632 (2018). 